\title{
THE SCATTERING MATRIX OF RANDOMLY ORIENTED INFINITE CYLINDERS
}

\author{
P. STAMMES \\ $E S A / E S T E C$ \\ P.O. Box 299 \\ NL-2200 AG Noordwijk \\ The Netherlands
}

Space Science Department and

ABSTRACT. A method is outlined to compute the scattering matrix of an ensemble of infinite cylinders in random orientation, as an approximation to the scattering matrix of an ensemble of very long but finite cylinders. Numerical checks are presented, which show that the results for infinite cylinders agree with theoretical results for very thick and very thin cylinders, as well as with numerical data for prolate spheroids and short finite cylinders.

\section{Introduction}

The scattering matrix of small particles is an important quantity in analyzing observations of sunlight scattered by interplanetary dust, because it completely describes the light scattering process including polarization. Often spheres have been considered as scattering models for interplanetary dust particles, since exact solutions of scattering by nonspherical particles are rare. The infinitely long circular cylinder is one of the few nonspherical shapes for which an exact analytical solution of Maxwell's equations is known. However, it poses some problems for use as a scattering model because of its infinite length. We have tried to overcome these problems in order to obtain a valid limit for a strongly elongated particle. The basic principle is that we consider a unit length of the infinite cylinder in all orientations.

In the past much research has been devoted to obtain the mathematical formulation for scattering by an infinite cylinder in arbitrary orientation with respect to the incident light (see e.g. [1-8]). In almost all previous work, however, scattering by only one cylinder was considered, or only the cross-sections for scattering and extinction were discussed. Here I will consider the scattering matrix of an ensemble of randomly oriented infinite cylinders, as an approximation to the scattering matrix of an ensemble of very long, but finite, cylinders.

\section{Definition of the scattering matrix}

We use the Stokes parameters $I, Q, U, V$ to describe a beam of light. $I$ is the intensity (in $\mathrm{W} / \mathrm{m}^{2}$ ) and $Q, U, V$ contain the polarization information of the beam. We consider scattering by an ensemble of particles which are randomly oriented. The scattering properties of the ensemble are completely contained in the $4 \times 4$ scattering matrix $\mathbf{F}(\Theta)(\Theta$ is the scattering angle), which transforms the Stokes parameters of the incident beam into those of the scattered beam. For particles that have a plane of symmetry, like cylinders, the scattering 
matrix of the ensemble has the form [2]

$$
\mathbf{F}(\Theta)=\left(\begin{array}{cccc}
F_{11}(\Theta) & F_{12}(\Theta) & 0 & 0 \\
F_{12}(\Theta) & F_{22}(\Theta) & 0 & 0 \\
0 & 0 & F_{33}(\Theta) & F_{34}(\Theta) \\
0 & 0 & -F_{34}(\Theta) & F_{44}(\Theta)
\end{array}\right)
$$

For unpolarized incident light, $F_{11}(\Theta)$ gives the intensity and $-F_{12}(\Theta) / F_{11}(\Theta)$ the degree of linear polarization of the scattered light.

\section{Procedure to obtain the scattering matrix}

We consider an infinitely long, homogeneous, circular cylinder with radius $\rho$, which is tilted with respect to the incident radiation (a plane wave). The angle of incidence, i.e. the angle between the incident beam and the cylinder normal, is $\alpha$. The scattered radiation propagates along the surfaces of cones, with apex angles $\pi-2 \alpha$ (cf. [4]).

The expression for the radiation scattered by one tilted infinite cylinder, for arbitrarily polarized incident radiation, is given by, e.g., [5-8]. We have to correct this expression for the infinite length of the cylinder using a factor $\cos \alpha$, in order to approximate a very long but finite cylinder. An infinite cylinder receives the same amount of light in any position, whereas a tilted finite cylinder receives an intensity reduced by a factor $\cos \alpha \operatorname{compared}$ to a perpendicular finite cylinder (cf. [8]).

To find the scattering matrix of an ensemble of infinite cylinders in random orientation, we have to integrate over all cylinder orientations in 3D-space. However, because of the scattering cone for one cylinder, we only have to integrate over those cylinders which are in the bisection plane of the directions of incidence and scattering. The resulting scattering matrix depends on: (i) the size parameter of the cylinder, $x=2 \pi \rho / \lambda$, where $\lambda$ is the wavelength, and (ii) the refractive index $m$.

\section{Checks}

To check our numerical results for the scattering matrix of an ensemble of randomly oriented infinite cylinders, we performed the following comparisons.

(a) The scattering matrix elements obeyed four general inequalities [9].

(b) We compared with Fraunhofer diffraction, which is the limit for very thick $(x \gg 1)$ cylinders. In Figure $1 F_{11}(\Theta)$ is shown for the exact case of infinite cylinders with $x=50$ and $m=1.31-0.1 i$, and for the case of Fraunhofer diffraction [10] with $x=50$. The agreement is good at small scattering angles but degrading at larger angles, as is expected. (c) We compared with Rayleigh-Gans scattering, which is the limit for "soft" and thin cylinders $(|m-1| \ll 1$ and $2 x|m-1| \ll 1)$. Figure 2 shows the excellent comparison between the exact result and Rayleigh-Gans theory [2] for $F_{11}(\Theta)$ in the case $x=0.1$ and $m=1.1$. The other matrix elements divided by $F_{11}$ compare equally well, their shape being that of ordinary Rayleigh scattering. Here we used a correction factor $1 / \cos \alpha$ in the Rayleigh-Gans theory of finite cylinders to make it applicable to infinite cylinders.

(d) We compared with computational results for short finite cylinders and prolate spheroids, obtained by the T-matrix method [11]. In Figure 3 the scattering matrix is shown for ensembles of infinite cylinders, finite cylinders and prolate spheroids, all having $m=1.31$. The size parameter of the infinite cylinders is $x=2.5$. As size parameter for the finite cylinders 


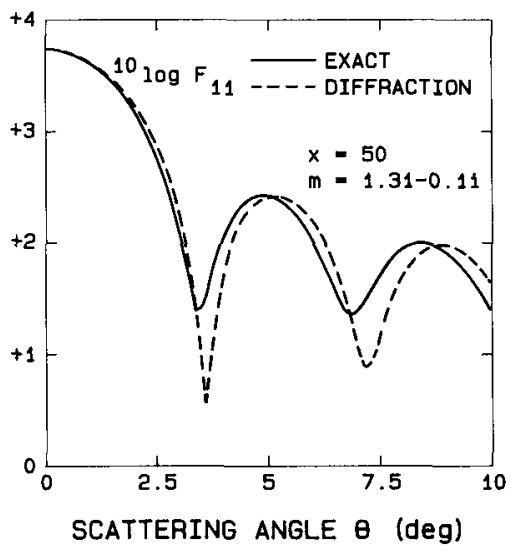

Figure 1. The scattering function $F_{11}(\Theta)$ for an ensemble of infinite cylinders in random orientation, following from exact theory and Fraunhofer diffraction.

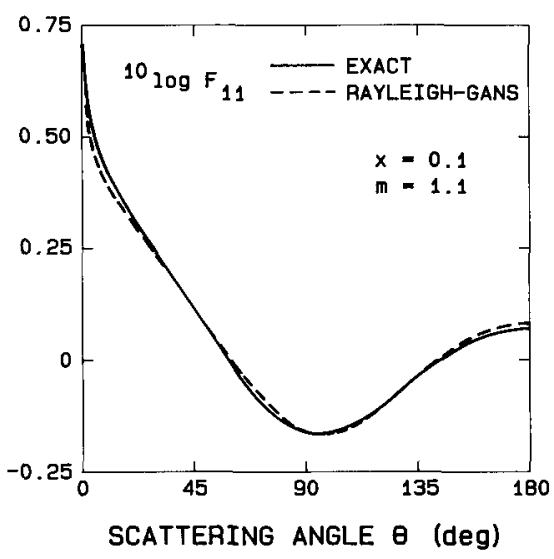

Figure 2. $F_{11}(\Theta)$ for an ensemble of infinite cylinders, following from exact and Rayleigh-Gans theory. For the RayleighGans results we used $2 \pi L / \lambda=10^{4}$, where $L$ is the cylinder length.
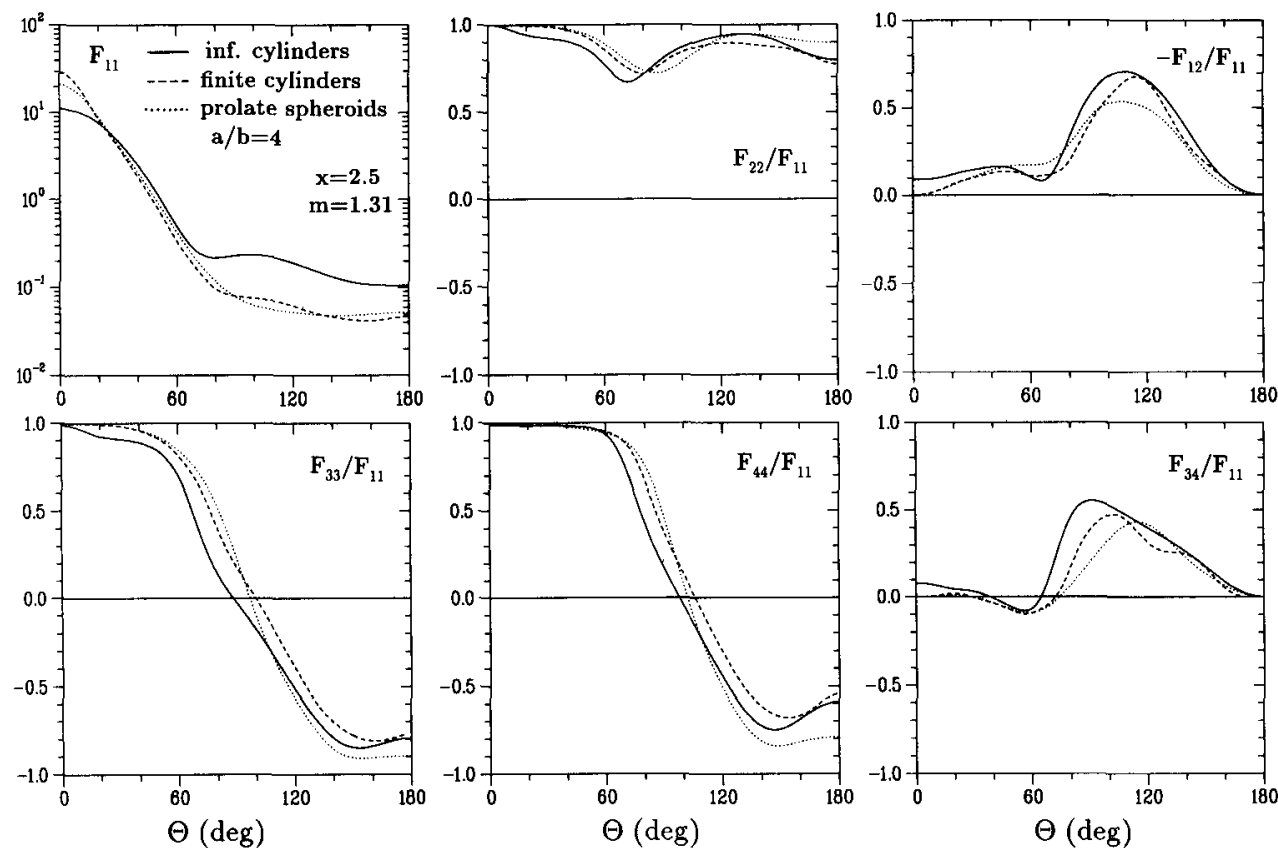

Figure 3. The scattering matrices of ensembles of infinite cylinders; finite cylinders and prolate spheroids. The finite cylinders and prolate spheroids both have axes ratio $a / b=4$. The results for finite cylinders and spheroids have been obtained with the T-matrix method. 
and prolate spheroids we take the size parameter of their semi-minor axis $b$, so $x=2 \pi b / \lambda$. The finite cylinders and prolate spheroids both have $x=2.5$ and axes ratio $a / b=4$. Almost all features in the scattering matrix elements of the finite cylinders and prolate spheroids are also found in the infinite cylinder results. If absorption is added the agreement improves.

\section{Conclusions}

It is possible to obtain the scattering matrix of an ensemble of randomly oriented infinite cylinders. Numerical tests have been performed to check the reliability of the results.

The presented solution for infinite cylinders is useful as an approximation to the scattering matrix of an ensemble of very long finite cylinders. Until now, it is not possible to obtain the scattering matrix of very elongated particles with other computational methods. The T-matrix method is limited to short cylinders and spheroids (axes ratio $\approx 4: 1$ ) and to size parameters (of the semi-major axis) of $\approx 15$. Our infinite cylinder solution works well up to much larger (radius) size parameters of $\approx 100$.

Some general characteristics of the scattering matrix of infinite cylinders are:

- The steepness of $F_{11}$ is much less than for short finite cylinders due to the absence of a strong forward peak. However, details in $F_{11}$ of short finite cylinders appearing at larger $\Theta$ also appear for infinite cylinders.

- Despite the fact that $F_{11}$ does not agree in magnitude with short finite cylinder results, the matrix element ratios $F_{i j} / F_{11}$ agree very well.

- The matrix element ratios $-F_{12} / F_{11}$ and $F_{34} / F_{11}$ tend to a non-zero value for $\Theta \rightarrow 0$. This differs from the behaviour for randomly oriented finite particles. The reason is the integration over the bisection plane to obtain the scattering matrix of randomly oriented infinite cylinders. This plane acts as a plane of preference for the polarization.

\section{Acknowledgements}

I am grateful to F. Kuik for performing the T-matrix method calculations, using a program from P. Barber (Clarkson Univ., Potsdam, USA), and acknowledge helpful discussions with J.W. Hovenier. This research has been supported by ESA's Research Fellowship Programme.

\section{References}

1. Wait, J.R. (1955) Can. J. Phys. 33, 189

2. Van de Hulst, H.C. (1957) "Light Scattering by Small Particles", J. Wiley \& Sons, New York; also Dover, New York, 1981

3. Lind, A.C. and Greenberg, J.M. (1966) J. Appl. Phys. 37, 3195

4. Kerker, M. (1969) "The Scattering of Light and Other Electromagnetic Radiation", Academic Press, New York

5. Liou, K.-N. (1972) Appl. Opt. 11, 667

6. Cohen, A. and Acquista, C. (1982) J. Opt. Soc. Am. 72, 531
7. Bohren, C.F. and Huffman, D.R. (1983) "Absorption and Scattering of Light by Small Particles", J. Wiley \& Sons, New York

8. Barabás, M. (1987) J. Opt. Soc. Am. A, 4, 2240

9. Hovenier, J.W., Van de Hulst, H.C. and Van der Mee, C.V.M. (1986) Astron. Astrophys. 157, 301

10. Takano, Y. and Tanaka, M. (1980) Appl. Opt. 19,2781

11. Barber, P. and Yeh, C. (1975) Appl. Opt. 14, 2864 